\title{
Belphégor
}

\section{Sérialité et personnages}

Du roman populaire à la bande dessinée

\section{Olivier Odaert}

\section{(2) OpenEdition \\ Journals}

Electronic version

URL: http://journals.openedition.org/belphegor/777

DOI: 10.4000/belphegor.777

ISSN: 1499-7185

Publisher

LPCM

\section{Electronic reference}

Olivier Odaert, « Sérialité et personnages », Belphégor [Online], 14 | 2016, Online since 17 October 2016, connection on 23 April 2019. URL : http://journals.openedition.org/belphegor/777 ; DOI : 10.4000/ belphegor.777

This text was automatically generated on 23 April 2019.

\section{(c)}

Belphégor est mis à disposition selon les termes de la Licence Creative Commons Attribution - Pas d'Utilisation Commerciale - Pas de Modification 4.0 International. 


\title{
Sérialité et personnages
}

\author{
Du roman populaire à la bande dessinée
}

\section{Olivier Odaert}

1 La bande dessinée moderne est née dans les pages des journaux de grande consommation et son expression a donc été contrainte, depuis ses origines, ou presque, par les formats de l'imprimé de masse qui, conjugués à la périodicité des supports médiatiques, imposèrent au genre naissant une logique de production sérielle. Certains précurseurs avaient publié des volumes monographiques au XIX ${ }^{e}$ siècle, comme en attestent les premiers " romans en estampes » ${ }^{1}$ de Rodolphe Töpffer, qui ont été diffusés sous la forme de livrets indépendants avant 1850. Par ailleurs, certains des comics les plus populaires seront publiés sous la forme d'albums dès les années 1910, même s'il s'agira longtemps de simples recueils de fragments. Mais au début du $\mathrm{XX}^{\mathrm{e}}$ siècle, lorsque les textes de l'histoire illustrée sont entrés dans l'image, et que la bande dessinée en vignettes, telle qu'elle se pratique encore aujourd'hui, a envahi les périodiques d'Amérique du Nord et d'Europe, la sérialité s'est imposée comme une loi du genre.

2 C'est pourquoi les unités narratives de la bande dessinée sont des unités formelles : à l'exception notable de la case ou vignette, qui ne constitue toutefois pas en soi de la bande dessinée, c'est-à-dire de la narration visuelle en séquences ${ }^{2}$, les ensembles de cases que constituent le strip ou la bande, la page complète et enfin, pour l'Europe, l'album cartonné - le $48 \mathrm{CC}$ de J.-C. Menu ${ }^{3}$-, ces unités graphiques et matérielles sont devenues les unités de sens autonomes, en quelque sorte, les chapitres et les paragraphes de la bande dessinée, parce qu'elles sont ses moyens de diffusion premiers et principaux.

Il faut d'ailleurs noter que la progressive autonomisation du genre et l'apparition d'éditeurs et de circuits de diffusion propres, au cours du $\mathrm{XX}^{\mathrm{e}}$ siècle, n'ont pas fondamentalement modifié cette configuration originelle de la syntaxe de la bande dessinée, et que les produits qui échappent à la structure en gigogne case/bande/album, imposée à l'origine par l'appareil médiatique, sont le plus souvent catalogués par les lecteurs et les auteurs, mais également par les théoriciens, comme des graphic novels ou romans graphiques ${ }^{4}$, précisément parce qu'ils expérimentent des formes de narration qui subvertissent formellement le modèle original en supprimant les bandes, en rompant 
l'unité sémantique de la page, ou encore en excédant largement la taille traditionnelle des albums.

4 En somme, la segmentation matérielle des récits de bande dessinée impliquée par les formats des imprimés de masse aura contribué à définir l'économie narrative du genre en imposant aux auteurs de produire des unités de sens de tailles fixes, qui soient sémantiquement autonomes, mais dont l'appartenance à un ensemble plus vaste soit tout aussi facilement repérable, afin de permettre au lecteur d'entrer dans la logique sérielle du récit. Les auteurs ont de ce fait progressivement mis en place toute une série de moyens pour répondre à la double injonction d'originalité et de répétition intimée par l'industrie culturelle.

Chronologiquement, les premiers moyens de la reconnaissance sérielle programmée par les comics ont été ceux de toute narration en série : comme les romans-feuilletons et les histoires illustrées, les bandes quotidiennes et les pages dominicales, qui étaient confinées dans un lieu dédié du périodique, faisaient appel à la mémoire du lecteur en systématisant le retour des personnages. Dans les premières décennies du $\mathrm{XX}^{\mathrm{e}}$, ces outils fondamentaux de la sérialité vont être appuyés par une affirmation croissante de la signature visuelle des auteurs. Winsor McCay, par exemple, va mettre en place un univers graphique cohérent dont la singularité ne se limite pas au réalisme fantastique du dessin, mais repose aussi sur toute une série de détails apparemment anodins, comme la typographie des titres, le lettrage, les gouttières entre les vignettes, la colorisation, soignée et contrastée, d'arrière-fonds chargés et variés, l'ensemble conférant aux pages de cet auteur une identité forte et distinctive ${ }^{5}$. Cette logique de différenciation, qui tendait à distinguer les productions en accentuant leurs singularités, et donc à favoriser la reconnaissance sérielle en insistant sur la figure auctoriale, sera toutefois le plus souvent contrebalancée par le marquage générique ou plutôt sous-générique des produits. En d'autres termes, et à quelques exceptions près, l'originalité stylistique des premiers auteurs de comics sera contrainte et contenue par la nécessité d'expliciter stylistiquement le genre de leurs séries.

6 Pierre Fresnault-Deruelle a proposé de classer les premiers comics des quotidiens américains en quatre sous-ensembles d'après leurs thèmes, et distingue de la sorte les "strips dramatiques», qu'il subdivise en «séries sentimentales» et "séries d'aventures ", des «strips humoristiques ", qu'il répartit en " séries de type satirique » et "séries humoristiques de type classique ${ }^{6}$. Cohérente sur le fond, et valide sur le long terme, cette typologie est aussi prégnante stylistiquement. Entre 1895 et 1934, les comics strips que Fresnault-Deruelle qualifie d'«humoristiques» dominent le secteur. S'ils mettent à peu près tous en scène des personnages stéréotypés qui incarnent la famille nucléaire type dans une satire sociale plutôt convenue, dont les personnages obligés sont le père buveur et joueur, la mère autoritaire et rébarbative, la jeune fille à marier, le soupirant et le garçon farceur, leur style n'est pas uniforme, qui dépend étroitement du sous-genre du récit, qu'il soit de «type classique » ou de «type satirique». Le premier pôle, le plus sage, se distingue par la lisibilité et le figement du dessin, qui associe à un trait le plus souvent clair et appuyé un cadrage théâtral. Les strips quotidiens de Winnie Winkle the Breadwinner que Martin Branner signe à partir de 1920 pour le Chicago Tribune Syndicate illustrent cette tendance du genre humoristique: leur précision descriptive appelle un humour de caractère, qui fait incontestablement écho au dessin de presse de la fin du XIX siècle tandis que la narration et l'articulation du récit y sont encore majoritairement de la responsabilité du texte qui, bien qu'intégré dans des ballons, porte 
le plus souvent seul la charge comique des fragments quotidiens. À l'autre pôle du genre humoristique, le strip satirique, plus exubérant, sert un humour visuel qui repose sur des mises en scènes plus spectaculaires, des personnages plus caricaturaux, et un dessin moins réaliste mais plus expressif et surtout plus dynamique. Ce style, visiblement marqué par l'influence du précurseur allemand Wilhelm Bush et importé aux États-Unis par Rudolph Dirks et ses Katzenjammer Kids dès 1912, deviendra la signature du genre de comics que pratiqueront notamment Cliff Sterret avec Polly and her Pals, dès 1912, et Billy DeBeck avec Barney Google, dès 1919.

7 Il faut insister sur le fait que ces variations dans le style des strips humoristiques, si elles peuvent être emblématisées par certaines séries, ne constituent pas des exemples de singularisation du style des auteurs : le type stylistique des séries dépend plus strictement de leur appartenance générique, qui ne laisse qu'une étroite marge de manœuvre à l'auteur pour imposer sa patte. La signature graphique de Martin Branner et Chic Young, par exemple, variera sensiblement selon qu'ils dessineront des strips en quelques cases ou des pages complètes en couleur: dans les séquences courtes des quotidiens, le comique langagier prédominera, dans un style de dessin statique et classique, tandis que dans leurs Sunday pages, les deux auteurs produiront des séquences axéez sur le dynamisme des personnages et tablant sur un humour visuel, à destination d'un lectorat plus diversifié que celui des quotidiens, et avec un style plus satirique et caricatural. Fred Opper, cet autre pionnier, qui avait développé un dessin de presse assez classique dans ses histoires illustrées pour le magazine Puck, au dix-neuvième siècle, adoptera lui aussi un humour résolument visuel et un style plus caricatural dans ses productions ultérieures, et notamment dans le satirique Happy Hooligan, à partir de 1900.

Cette loi des genres vaudra aussi, quelques décennies plus loin, pour les «séries dramatiques ». Après que Chester Gould et Alex Raymond eurent inventé le genre du comics d'aventures en s'appropriant les figures les plus marquantes de la fiction de grande consommation, comme le détective ou l'espion, dans les séries Dick Tracy, à partir de 1931, ou Secret Agent X-9, à partir de 1934, leur style de dessin sera repris par leurs émules au même titre que leurs thèmes, ce qui confirme l'existence d'une signature visuelle des genres, qui fonctionne comme moyen d'inscription générique autant que sérielle, au moins dans la première moitié du XXe siècle. Kurt Caesar, par exemple, lorsqu'il signera, à partir de 1937, la série Will Sparrow, qui paraîtrait dans le Journal de Spirou à partir de 1938 sous le titre de Bill l'Albatros, s'inspirera visiblement du dessin tout autant que des sujets de prédilection d'Alex Raymond. Le développement de la bande dessinée d'aventure est en ce sens un bouleversement stylistique autant que thématique, qui témoigne par ailleurs d'une influence grandissante du cinéma sur la narration visuelle.

On voit donc comment la contrainte de lisibilité des genres, qui imposait aux auteurs de se conformer à un style prédéfini par leur genre d'expression, s'opposait diamétralement à la contrainte de visibilité des séries, qui devaient se démarquer un tant soit peu pour exister, et inhibait les caractéristiques distinctives des dessinateurs. Cette logique d'uniformisation va amener les premiers auteurs et les premiers éditeurs de bande dessinée à développer superlativement les ressorts plus traditionnels de la sérialité, affaiblis par ce nivellement stylistique généralisé.

Leur stratégie de distinction la plus visible va porter sur l'unité graphique et narrative de tout récit de bande dessinée: le personnage. Le retour des personnages était, depuis l'origine du genre, une condition presque sine qua non de la sérialité. Le Yellow kid et Buster Brown chez Outcault, les Katzenjammer kids chez Dirks, ainsi que tous les autres 
protagonistes et systèmes de personnages mis en scène par Opper, Sterret, Branner et tant d'autres assumaient l'essentiel de la cohérence narrative des séries, mais ne contribuaient pas pour autant à leur différenciation d'avec leurs concurrentes. Ces différents personnages, en effet, hérités de la comédie de boulevard, pouvaient être ramenés à quelques types dont les plus traités ont sans doute été le petit garçon malicieux (Buster Brown, Perry Winkle, etc.) et la jeune femme responsable et élégante à la fois (Winnie Winkle, Blondie, etc.), archétypes repris et développés pendant des décennies des deux côtés de l'Atlantique, avec de multiples variantes. Aussi, quand cette similarité participait de la lisibilité du genre du comic humoristique dans sa totalité, elle a pu constituer un obstacle à la sérialité en produisant un effet de confusion que les auteurs ont contrebalancé en insistant jusqu'à la démesure la plus totale sur la personnalité graphique de leurs protagonistes.

11 Là encore, aux origines du genre, les productions de Winsor McCay constituent un précédent innovant et annonciateur des logiques futures, de même que les planches de celui que l'on désigne souvent comme l'«inventeur» de la bande dessinée moderne, Richard Felton Outcault. Dès les années 1900, les auteurs de Little Nemo et du Yellow Kid parieront en effet sur des procédés qui seront progressivement utilisés par la plupart des auteurs de comics. Premièrement, Oucault et McCay vont progressivement ajouter à leur appareil graphique un titre hypertrophié qui intègrera le nom du personnage principal au récit en images. La plupart des comics étaient déjà signalés par un titre assez grand reprenant le nom d'un ou plusieurs personnages ou encore annonçant, sans raison apparente, puisque les pages parlaient d'elles-mêmes, la section des strips, mais ces intitulés ne faisaient pas à proprement parler partie du récit, qu'ils surplombaient. Avec Outcault et McCay, les titres, comme les paroles des personnages, seront intégrés par l'image, et participeront de sa logique. Les titres de Little Nemo, par exemple, sont à la fois typographiquement redondants, et donc identifiables en tant qu'images tout autant qu'en tant que langage, mais sont également adaptés aux couleurs de l'histoire, et donc singularisés en fonction du récit. Plus tard, de nombreux auteurs de comics d'aventure au long cours s'approprieront ce mode de signalisation, et notamment Chester Gould avec Dick Tracy.

Ensuite, et ce procédé se généralisera, Outcault et McCay avaient dès le début de leurs séries trouvé un moyen simple et efficace de donner à leurs personnages une identité graphique distinctive. Le Yellow Kid est assimilé à son éternelle chemise jaune ; Buster Brown est particularisé par son costume marin, que l'on désigne désormais comme le Buster Brown suit ; Nemo, enfin, est un rêveur qui se réveille à la fin de chaque planche et qui, dans ses rêves comme à son réveil, porte le plus souvent une longue chemise de nuit blanche contrastant avec les couleurs chatoyantes qui l'entourent, et le met donc en évidence tout en lui assurant une identité graphique stable. Il faut d'ailleurs noter que lorsque Nemo porte un autre vêtement, son travestissement est généralement mis en scène, ce qui surdétermine la valeur métonymique de sa chemise fétiche, et annonce le thème du déguisement, qui sera central dans les bandes dessinées des années 1930 . Ce traitement vestimentaire de l'identité des personnages n'est pas neuf à l'époque de McCay et Outcault : Dirks avant eux, l'avait déjà annoncé dans les Katzenjammer kids, mais il revient à ces auteurs d'avoir fait de l'identité vestimentaire de leurs personnages l'élément premier de l'identification de leurs séries.

13 Ce mode de différenciation graphique des personnages, développé par Outcault et McCay, va devenir crucial dans les années 1930 et se renforcer avec d'autant plus de vigueur que 
la multiplication des séries appellera des identités sans cesse plus fortes. Chester Gould, à partir de 1931, fera du borsalino jaune un emblème sans équivoque du personnage de Dick Tracy. En 1938, des deux côtés de l'Atlantique, deux personnages vont mener cette logique à son comble en se confondant littéralement avec leur costume: Spirou, en Belgique, et Superman, aux États-Unis. Le premier, créé par Rob-Vel en avril 1938, a gardé jusqu’à aujourd'hui son costume de groom au Moustique hôtel, qui n'aura pourtant répondu de son occupation professionnelle que le temps de quelques planches. Quant au second, créé quelques années plus tôt par Joe Shuster et Jerry Siegel, il apparaîtra dans Action Comics à partir de juin 1938 déjà équipé de son célèbre costume moulant, qui allait, avec quelques autres, et notamment celui du Phantom de Lee Falk (1936), servir de modèle vestimentaire à la plupart des super-héros de la bande dessinée américaine ${ }^{7}$.

Un autre développement majeur du genre, que McCay avait également anticipé, et qui peut se concevoir comme une autre résultante de la même nécessité d'accrocher et de fidéliser les lecteurs dans un contexte où la lisibilité du genre doit toutefois rester immédiate, sera l'allongement des récits, qui vont former à l'intérieur des séries des unités narratives plus longues, articulées en plusieurs dizaines de pages et livrées, selon les cas, par strips, par fractions de pages ou par pages entières, et par la suite, plusieurs pages à la fois avant, in fine, de paraître sous la forme d'albums indépendants. Ce n'est pas un hasard si cette évolution est concomitante de développement de la bande dessinée d'aventures, dont les récits, comme en atteste assez clairement l'évolution de la série des Aventures de Tintin, publiées par Hergé à partir de 1929, vont progressivement passer de la simple suite de péripéties vaguement organisées autour d'un thème commun, comme le voyage Au pays des soviets (1930), à des histoires à intrigue plus cohérentes, dont la portée dépassera d'ailleurs assez rapidement le format de l'album, ainsi qu'en atteste l'exemple du Lotus Bleu (1936), qui fait directement suite aux aventures des Cigares du pharaon (1934).

Les auteurs vont, dans ce contexte, délaisser les personnages traditionnels de la bande dessinée, dont les frasques peuplaient les journaux depuis plusieurs dizaines d'années déjà, et trouver une inspiration nouvelle dans le roman populaire sériel, dont ils vont réactiver les figures les plus marquantes, celles du détective, du justicier ou encore du reporter. On connaît la théorie d'Umberto Eco à ce sujet, qui consiste à faire de Superman un héritier du comte de Monte-Cristo et, en passant, des personnages de la culture populaire des modèles $d u$ fascisme ${ }^{8}$. Indépendamment de l'aspect idéologique de ce commentaire, il faut reconnaître que les personnages de bande dessinée qui ont émergé dans les années 1930, et pas seulement les super-héros, constituent pour la plupart des emprunts au roman populaire, qui avait développé depuis longtemps des stratégies de narration sérielle que la bande dessinée allait partiellement s'approprier en reprenant ses figures les plus marquantes, dont le codage déjà ancien constituait d'ailleurs un gage de lisibilité.

Mais la reprise de ces personnages, aussi évidente, et parfois même grossière fut-elle, allait devoir s'accommoder des spécificités du genre et partant, des contraintes sérielles propres à la narration en images. Pierre Fresnault-Deruelle et les autres spécialistes de la bande dessinée qui, à sa suite, ont contribué à décrire et à théoriser le genre dans une perspective sémiologique, se sont généralement concentrés sur les problèmes d'intégration du texte et de l'image, avec pour objectif explicite de démontrer la responsabilité narrative des images, mais n'ont accordé aux personnages que très peu d'attention. Pourtant, et en dépit de son inscription claire dans la tradition de la culture médiatique, la fonction narrative et sémiologique du personnage de bande dessinée 
constitue une des spécificités distinctives du genre, ce qui apparaît très clairement au regard des descriptions classiques du personnage de roman, des formalistes russes à Vincent Jouve, en passant par Philippe Hamon.

Dans L'Effet-personnage dans le roman, son essai de 1992, Vincent Jouve énonce par exemple cette évidence qu'« un roman où chaque personnage serait entièrement décrit des pieds à la tête [...] à chacune de ses apparitions serait non seulement oiseux, mais illisible. " ${ }^{9} C^{\prime}$ 'est pourtant la gageure à laquelle tous les auteurs de bande dessinée doivent s'affronter à chaque case. Jouve ajoute plus loin, dans un vocabulaire dont les connotations ne sont en l'occurrence pas anodines, que « [l'auteur] laisse le lecteur remplir de lui-même les cases manquantes. Les actions d'un personnage n'ont pas besoin d'être décomposées étape par étape $"{ }^{10}$ Or, en bande dessinée, c'est bien cette décomposition des actions du personnage qui constitue la trame narrative, comme en attestent les très nombreuses bandes dessinées sans décor, et parfois même sans texte.

Cette différence fondamentale entre le statut sémiologique des personnages de bande dessinée et de roman explique toute une série de développements que les modèles archétypaux issus de la littérature populaire vont connaître dans les cases des bandes dessinées. La nécessité d'expliciter visuellement les états d'âme des personnages va ainsi donner lieu à toute une syntaxe graphique et corporelle de l'intériorité, qui passera par une exagération théâtrale des mimiques et des gestes, mais aussi par une attention accrue à l'aspect graphique des héros et donc, à leur garde-robe, ce qui peut expliquer, en plus des facteurs idéologiques mentionnés par Eco, et de la contrainte sérielle de singularisation visuelle des héros, la surenchère des comics américains, dont les superhéros, avant d'être des êtres fantastiques, sont d'abord des personnages, dont les costumes aux couleurs criardes et les acrobaties surhumaines ne sont au fond que les épithètes superlatifs.

Mais le développement de la bande dessinée d'aventure, lié à l'émancipation éditoriale du genre et à l'allongement des récits, en suscitant une importation massive des modèles héroïques du roman populaire, allait amener les auteurs à s'approprier une partie non négligeable des pratiques narratives de la littérature de masse. L'exemple emblématique de la bande dessinée européenne, celui de Tintin, illustre ce progressif rapprochement.

20 Les premières aventures du jeune reporter, publiées dans Le Petit Vingtième à partir de 1929, et en album dès 1930, constituent déjà un récit au long cours, quoique constitué par une suite d'épisodes simplement juxtaposés, dont l'articulation avec l'intrigue générale reste tout à fait sommaire, voire accessoire. Leur protagoniste, Tintin, est une copie presque conforme d'un des personnages les plus célèbres de la littérature populaire du premier vingtième siècle, Joseph Rouletabille, le fameux héros de Gaston Leroux. Outre un surnom passablement ridicule, les deux héros partagent une même occupation professionnelle: ils sont reporters, et pratiquent tous deux ce métier à des fins plus policières que strictement journalistiques. Ils se distinguent aussi par leur très jeune âge, marqué notamment par un visage poupon. Leroux décrit Rouletabille comme un "petit reporter", un "gamin", un "petit garçon" qui «semblait avoir pris sa tête, ronde comme un boulet, dans une boîte à bille ${ }^{11}$, ce qui explique du reste son surnom. Quant à Tintin, son visage ovale est tout juste surmonté d'une houpette, et l'on pourrait presque ajouter, en poussant peut-être le rapprochement un peu loin, que ses sourcils exagérément courbés rappellent les deux mystérieuses bosses dont Leroux affuble son héros, et dans lesquelles il situe le secret de sa pénétrante sagacité. 
21 Mais au-delà de cette ressemblance de surface, ce sont les pratiques sérielles de Gaston Leroux qu'Hergé va progressivement s'approprier et adapter à la bande dessinée. Les deux auteurs mettent en pratique un certain nombre de trucs et ficelles assez courants, par exemple lorsque Leroux, dans Le Mystère de la chambre jaune, évoque déjà Le Parfum de la dame en noir, épisode suivant des aventures de son personnage, dans lequel il réitérera cet effet d'annonce en évoquant le départ de Rouletabille pour la Russie, mis en scène dans Rouletabille chez le tsar, titre qui capitalise d'ailleurs pour la première fois la notoriété naissante du petit reporter. Hergé, de la même manière, annoncera dans Tintin au Congo le futur voyage en Amérique, en confrontant son Tintin aux hommes d'Al Capone, effet qu'il réitérera dans Les Cigares du pharaon, en informant dès le départ de cette histoire que le but du voyage de Tintin est Shangaï, qu'il n'atteindra toutefois que dans l'album suivant, Le Lotus Bleu. Mais la filiation entre les deux auteurs, et donc entre les deux genres, se vérifie surtout à la comparaison de leurs techniques de sérialisation. La première concerne le système des personnages tandis que la seconde touche à la mise en intrigue.

La similitude la plus évidente entre les systèmes de personnages de Leroux et Hergé est que leurs protagonistes sont presque toujours accompagnés par un personnage secondaire, qui joue un rôle que Philippe Hamon a qualifié d'embrayeur ${ }^{12}$ et qui consiste, en l'occurrence, à représenter le lecteur dans le récit et donc à incarner une sorte d'interface entre le héros et lui, comme le fait le célèbre Dr Watson dans les aventures de Sherlock Holmes. Dans les aventures de Rouletabille, ce rôle revient à Sainclair, dans celles de Tintin, il appartient à l'increvable Milou. S'il peut apparaître quelque peu irrévérencieux de comparer l'avocat naïf de Leroux au petit chien pensant d'Hergé, il faut reconnaître que ces deux personnages servent principalement à commenter l'action du protagoniste, ce qui contribue à son héroïsation. Pour le dire simplement, quand Sainclair n'admire pas l'« intelligence si éveillée et si originale ${ }^{13}$ de Rouletabille, par rapport à laquelle il fait piètre figure, il rappelle au lecteur les tenants de l'intrigue. Milou, à peu de choses près, est chargé des mêmes fonctions de commentaire et de rappel.

Mais la particularité la plus sensible du système des personnages, dans les premières aventures de Rouletabille, est qu'il constitue une cellule familiale, comme le lecteur le découvrira progressivement. Dans le premier épisode, on comprend en effet que le criminel, qui n'est autre que Frédéric Larsan, l'enquêteur dépêché pour résoudre le Mystère de la chambre jaune, est en fait le père de Rouletabille, un célèbre malfrat nommé Ballmeyer. Dans Le Parfum de la dame en noir, Sainclair et le lecteur apprendront que $\mathrm{M}^{\text {lle }}$ Stangerson, la victime du premier livre, autrefois aimée de son bourreau, n'est autre que la mère du petit reporter. Cette structure a l'avantage d'être immédiatement identifiable par n'importe quel lecteur, tout en constituant un contingent de personnages qu'il sera aisé pour Leroux de mobiliser à nouveau dans d'autres textes. Elle répond donc aux exigences de reconnaissance et de répétition de la culture médiatique. Contrairement à Leroux, qui après avoir donné de la consistance à son héros en le situant dans l'histoire d'une famille, l'enverra faire la preuve de ses talents en Russie ou en Allemagne, Hergé commencera par laisser son héros voyager seul, avant de progressivement lui associer une série de personnages récurrents qui constitueront ce que l'on a coutume d'appeler la « famille hergéenne ». Les liens familiaux n'y sont pas réels, comme chez Leroux, mais on y retrouve des figures comparables à celles que Leroux met en scène: Le fameux professeur Tournesol, par exemple, scientifique fantasque mais habile, annoncé dès Les Cigares du Pharaon par Philémon Siclone, rappelle le professeur Stangerson, chercheur célèbre et grand-père de Rouletabille. 

récurrent, celui du travestissement, qui constituera le moteur narratif principal de ses romans à énigme. Dans Le Mystère de la chambre jaune, premier volume des aventures du reporter, par exemple, seule l'habileté au déguisement de Frédéric Larsan-BallmeyerJean Roussel, l'antagoniste, permet de justifier une enquête qui, sans cela, aurait été résolue avant d'avoir véritablement commencé : en se rendant sur les lieux du crime, Sainclair et Rouletabille rencontrent en premier lieu le coupable, qu'ils confondent cependant avec l'enquêteur. Par ailleurs, les deux « mystères » qui justifient le démarrage et la poursuite du récit, celui «de la chambre jaune», et celui de la "galerie inexplicable ", reposent sur la confusion entre, d'une part, les signes d'une agression et une agression réelle, et d'autre part, entre Frédéric Larsan et son double maléfique. C'est donc le travestissement des situations et des personnages qui permet non seulement à Leroux d'articuler les différents chapitres de ses romans, destinés à une publication périodique, mais aussi les différentes aventures de Rouletabille, et ce ressort narratif constitue donc un moyen privilégié de la sérialité de son œuvre à différents niveaux d'articulation.

Chez Hergé, le motif du travestissement sera, tout autant qu'il l'était chez Leroux, un moteur d'intrigue et un mode d'articulation des fragments et des volumes, mais aussi un des embrayeurs comiques les plus usités. Dès les premiers épisodes de ses aventures, en effet, Tintin et ses ennemis se déguisent à chaque page, entraînant des quiproquos sans fin. Et au moment de troquer les suites de gags et d'aventures pour une première intrigue de longue haleine, Hergé va utiliser ces procédés avec une régularité telle que l'on pourrait presque lire Les Cigares $d u$ pharaon comme une longue suite de malentendus, qui sont généralement des défauts d'identification. Tintin lui-même est confronté à une succession d'illusions : il s'emporte contre les acteurs du film tourné par Rastapopoulos, avant de se réjouir, dans le désert, à la vue d'un mirage. Le héros est ensuite mal identifié par toute une série de personnages, à commencer par les Dupond et Dupont. Enfin, ce tressage serré de malentendus sur l'identité, et donc sur la fonction narrative des personnages, culmine à la fin du volume, lorsque Tintin, déguisé une fois de plus, révèle l'identité des membres d'une société secrète, qu'il démasque, au sens propre comme au sens figuré.

En somme, et pour conclure, si le genre narratif de la bande dessinée a de toute évidence été très strictement configuré par les formats et la périodicité des imprimés de masse, la sérialité obligée des premiers temps semble avoir contraint la majorité des auteurs à adopter des styles génériques, c'est-à-dire lisibles, et partant à déléguer à l'apparence et au nom de leurs personnages l'identité de leurs produits, ce qui conduira à l'héroïsme superlatif des comics américains, et plus généralement à accentuer l'identité graphique des héros de bande dessinée. Mais paradoxalement, cette responsabilité des protagonistes des récits va conduire les auteurs à s'approprier les modèles du roman populaire, et partant, à engager une évolution du genre vers des récits plus longs et plus complexes. Cette situation, comme semble l'indiquer l'exemple d'Hergé, va conduire les dessinateurs à emprunter au roman populaire ses ficelles les plus efficaces et à faire d'un art jusqu'alors dédié aux farces et aux gags un genre narratif à part entière, dont il reste toutefois à mesurer dans le détail tout ce qu'il doit à la civilisation du journal. 


\section{NOTES}

1. Thierry SMOLDEREN, Naissances de la bande dessinée. De William Hogarth à Winsor McCay, Paris, Les Impressions nouvelles, 2009, p. 41.

2. Thierry GROENSTEEN affirme qu' ' il faut reconnaître comme unique fondement ontologique de la bande dessinée la mise en relation d'une pluralité d'images solidaires ». (Système de la bande dessinée, Paris, PUF 1999, p. 21.)

3. J.-C. MENU, Plates-bandes, Paris, L'Association (Éprouvette), 2005.

4. La distinction entre comics et graphic novel est d'abord sociologique : le roman graphique est un produit littéraire et élitiste, par opposition à la simple bande dessinée sérielle. Mais cette opposition se marque dans la production par une subversion des formes traditionnelles de la bande dessinée sérielle. Ed Tan considère par exemple que « comics may be called formulaic [...]. The classical formula [...] has not only been canonised as to genre, themes and (generic) iconography [...] but also as regards details of its lay-out ». Ed S. TAN, « The telling face in comic strip and graphic novel », dans Jan BAETENS (dir.), The Graphic novel, Leuven, Leuven University Press, 2001, p. 31.

5. L'influence des imageries de la maison Quantin sur les planches de Little Nemo in slumberland mise en évidence par Antoine Sausverd concerne le dessin et les thèmes, mais pas la signature graphique de cette série telle qu'elle est évoquée ici. Voir Antoine SAUSVERD, «Le Petit Lucien au Pays des rêves ", dans Töpfferiana, 24 novembre 2008. [En ligne] http://www.topfferiana.fr (Page consultée le $1^{\mathrm{er}}$ mai 2013).

6. Pierre FRESNAULT-DERUELLE, Récits et discours par la bande. Essais sur les comics, Paris, Hachette, 1977, p. 15.

7. Il faut noter que les aventures des héros de bande dessinée Flash Gordon (Alex Raymond, 1934) et Phantom (Lee Falk, 1936) ont été publiées avant celles de Superman, mais à ses débuts, Flash Gordon ne portait pas de costume particulier, tandis que Phantom n'a paru en couleurs qu'à partir de 1939.

8. Umberto ECO, De Superman au Surhomme (1978), trad. de l'italien par Myriem Bouzaher, Paris, Grasset, 2005.

9. Vincent JOUVE, L'Effet-personnage dans le roman, Paris, PUF, 1992 (Écriture), p. 28.

10. Ibid., p. 33.

11. Gaston LEROUX, Le Mystère de la chambre jaune. Première partie: Le drame du Glandier, Paris, Lafitte, 1920, p. 6, $11 \& 60$.

12. Philippe HAMON, «Pour un statut sémiologique du personnage », dans R. BARTHES e.a., Poétique du récit, Paris, Seuil, « Points », p. 144.

13. Gaston LEROUX, op. cit., p. 12. 


\section{ABSTRACTS}

En bande dessinée, le moyen privilégié de l'identification des séries est le personnage. À l'origine, le nom du ou des protagonistes tenait lieu de signature, tandis que le style du dessin servait seulement à indiquer au lecteur à quel sous-genre de bande dessinée il avait affaire. C'est pourquoi, dans les années 1910, quand la multiplication des séries va contraindre les auteurs à adopter des signatures visuelles plus distinctives, on n'assistera pas à une diversification stylistique, mais à une surenchère dans l'apparence graphique des personnages, aux garde-robes de plus en plus criardes et excentriques, comme en attestent les exemples de Spirou et Superman. Cet attrait nouveau pour des personnages (stéréo-)typés va pousser les dessinateurs à s'inspirer parfois très littéralement du répertoire du roman populaire, auquel ils emprunteront des types de héros prêts-à-porter, mais aussi des trucs et ficelles de la sérialisation, comme en témoigne l'exemple de Hergé qui, pour inventer Tintin, imitera Rouletabille, le fameux personnage romanesque de Gaston Leroux, auteur auquel il empruntera aussi les principaux ressorts de ses intrigues, et notamment le motif du déguisement.

\section{INDEX}

Mots-clés: Bande dessinée, Roman populaire, personnage, sérialité, Gaston Leroux, Hergé

\section{AUTHOR}

\section{OLIVIER ODAERT}

Olivier Odaert est chercheur post-doctoral à l'Université de Limoges. Ses travaux portent principalement sur la littérature d'expression française de la première moitié du vingtième siècle, dans les genres du roman, du reportage et de la bande dessinée. Il a publié de nombreux travaux scientifiques sur ces sujets, et en particulier sur l'œuvre d'Antoine de Saint-Exupéry, dont « Une résistance littéraire » (Cahiers de la NRF, 2013) et « Le Mythe du Petit Prince » (Gallimard, 2013). En 2012, il a soutenu une thèse de doctorat intitulée "Saint-Exupéry écrivain », à l'Université de Louvain (Belgique). 